\title{
AN APPROXIMATE THEORY FOR THE DYNAMIC BEHAVIOR OF SOAP FILMS*
}

\author{
BY \\ R. W. DICKEY \\ University of Wisconsin, Madison
}

1. Introduction. The purpose of this paper is to study the dynamic behavior of a soap film which is stretched between two coaxial rings (cf. Fig. 1.1a). An "exact" theory for the axially symmetric deformation of such films has been described in [1]. This theory leads to a pair of nonlinear, hyperbolic partial differential equations with the axial and radial displacements from equilibrium as the quantities to be determined. Unfortunately these equations are rather complicated; thus the study of the equations has been limited to numerical approximations of the solutions (cf. [1]).

In this paper we will describe an approximate theory for the axially symmetric deformation of soap films which leads to a great simplification of the governing equations. This theory is based on two approximations which, while new to the theory of soap films, are not unusual in the study of elastic membranes and strings. In order to describe the approximations it is convenient to introduce the notation $u(x, t)$ and $w(x, t)$ for the axial and radial displacements of the film from some appropriate surface connecting the two end rings (e.g. the equilibrium surface). We will assume that $u_{x}(x, t)$ is small of first order. No assumption will be made on the magnitude of $w(x, t)$ or its derivatives. This approximation was first introduced by Foppl [2] in the study of plane membranes. Related approximations have been used by Bromberg and Stoker [3] and Reissner [4] in the theory of curved membranes. Secondly, we will assume that the kinetic energy due to axial motion can be neglected in comparison to the kinetic energy due to radial motion. This assumption is also quite typical in various special theories of elasticity (cf. $[5,6,7])$.
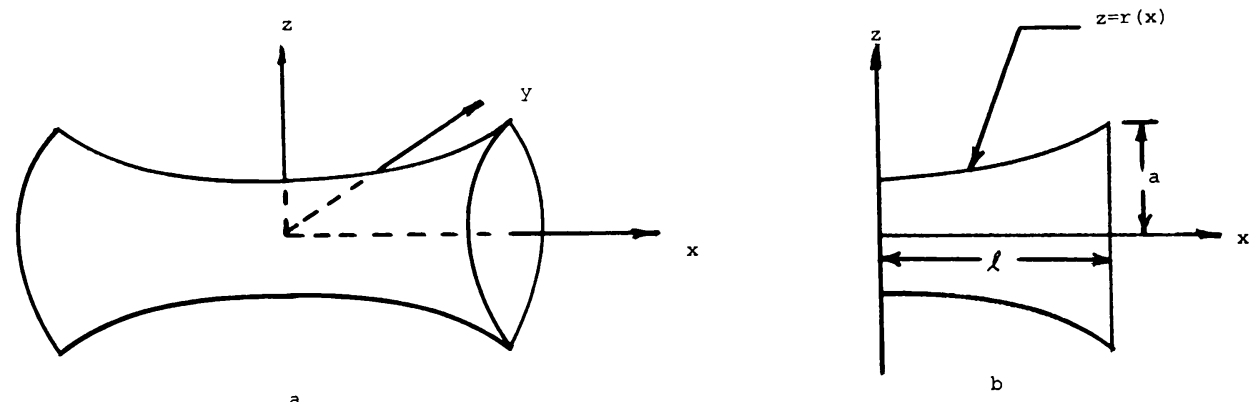

Fig. 1.1.

* Received February 25, 1981. This research was supported by the National Science Foundation, Grant No. 75-06366. 
In Sec. 2 it will be shown that the approximations indicated above lead to a single, nonlinear, partial integro-differential equation for the determination of $w$. In Sec. 3 the static problem is studied. It is shown that if $a / l$ (cf. Fig. $1.1 \mathrm{~b}$ ) is sufficiently large there are two equilibria (both are catenaries of revolution) and if $a / l$ is sufficiently small there are no equilibria connecting the end rings. There is a certain critical value of $a / l$, say $a / l=c$, for which there is exactly one equilibrium. These results agree with the exact theory of soap films described in [8]. In Sec. 4 it will be shown that if $a / l>c$ one of the catenaries is linearly (dynamically) stable. If $a / l=c$ the single equilibrium is unstable.

2. The dynamic equations. Two coaxial rings of radius $a$ are located at $x= \pm l$. We will assume that these rings are connected by a surface of revolution (the soap film) obtained by rotating a curve $z=r(x)$ about the $x$ axis. For simplicity we will assume that $z=r(x)$ is symmetric about the origin, i.e. $r(-x)=r(x)\left(r^{\prime}(0)=0, r(l)=a\right)$ and that this symmetry property is retained by film during any deformation. If the surface $z=r(x)$ is not an equilibrium surface or if the film is in motion at time $t=0$ a point of the film located at $(x, r(x))$ at $t=0$ will be at some new position $(x+u(x, t), r(x)+w(x, t))$ at some later time $t$. The surface area of this deformed surface will be given by

$$
A(t)=4 \pi \int_{0}^{l}(r+w)\left(\left(1+u_{x}\right)^{2}+\left(r^{\prime}+w_{x}\right)^{2}\right)^{1 / 2} d x
$$

$\left(r^{\prime}=d r / d x\right)$. The kinetic energy of the surface will be

$$
\text { K.E. }=4 \pi \int_{0}^{l} \frac{\rho}{2} r\left(1+\left(r^{\prime}\right)^{2}\right)^{1 / 2}\left(w_{t}^{2}+u_{t}^{2}\right) d x
$$

where $\rho$ is the surface density.

The theory of soap films assumes the potential energy of the film is proportional to the surface area, i.e.,

$$
\text { P.E. }=K A(t)
$$

where $K$ is a constant of proportionality. As was indicated in Sec. 1, it will be assumed that

$$
\begin{aligned}
\left|u_{x}\right| & \ll u_{x}^{2}, \\
\int_{0}^{l} \frac{\rho}{2} r\left(1+\left(r^{\prime}\right)^{2}\right)^{1 / 2} u_{t}^{2} d x & \ll \int_{0}^{l} \frac{\rho}{2} r\left(1+\left(r^{\prime}\right)^{2}\right)^{1 / 2} w_{t}^{2} d x .
\end{aligned}
$$

Thus we obtain the approximate expressions for the potential and kinetic energies

$$
\begin{gathered}
\text { K.E. } \approx 4 \pi \int_{0}^{l} \frac{\rho}{2} r\left(1+\left(r^{\prime}\right)^{2}\right)^{1 / 2} w_{t}^{2} d x, \\
\text { P.E. } \approx 4 \pi \int_{0}^{l}(r+w)\left(1+2 u_{x}+\left(r^{\prime}+w_{x}\right)^{2}\right)^{1 / 2} d x .
\end{gathered}
$$

The equations of motion for the film are simply the Euler equations (cf. [9]) for the integral

$$
\int_{0}^{t}(\text { K.E. }- \text { P.E. }) d t \text {. }
$$


These Euler equations are easily found to be

$$
\begin{gathered}
\frac{\partial}{\partial x}\left(\frac{(r+w)}{\left.\left(1+2 u_{x}+\left(r^{\prime}+w_{x}\right)^{2}\right)^{1 / 2}\right)}\right)=0 \\
\rho r\left(1+\left(r^{\prime}\right)^{2}\right)^{1 / 2} w_{t t}-K \frac{\partial}{\partial x}\left(\frac{(r+w)\left(r^{\prime}+w_{x}\right)}{\left(1+2 u_{x}+\left(r^{\prime}+w_{x}\right)^{2}\right)^{1 / 2}}\right)+K\left(1+2 u_{x}+\left(r^{\prime}+w_{x}\right)^{2}\right)^{1 / 2}=0 .
\end{gathered}
$$

The appropriate boundary conditions on $u(x, t)$ and $w(x, t)$ are

$$
u(0, t)=u(l, t)=0, \quad w_{x}(0, t)=w(l, t)=0 .
$$

The equations (2.7) are greatly simplified by the observation that (2.7a) implies that

$$
\frac{r+w}{\left(1+2 u_{x}+\left(r^{\prime}+w_{x}\right)^{2}\right)^{1 / 2}}=f(t)
$$

i.e., the quantity on the left of (2.9) is independent of $x$. Thus rewriting (2.9) in the form

$$
(r+w)^{2}=f(t)^{2}\left(1+2 u_{x}+\left(r^{\prime}+w_{x}\right)^{2}\right)
$$

it follows from (2.8a) that

$$
f(t)^{2}=N[r+w]^{2}=\frac{\int_{0}^{l}(r+w)^{2} d x}{\int_{0}^{l}\left(1+\left(r^{\prime}+w_{x}\right)^{2}\right) d x} .
$$

This result may be combined with (2.7b) to show that $w(x, t)$ must satisfy

$$
\rho r\left(1+\left(r^{\prime}\right)^{2}\right)^{1 / 2} w_{t t}-K N[r+w] \frac{\partial}{\partial x}\left(r^{\prime}+w_{x}\right)+\frac{K}{N[r+w]}(r+w)=0
$$

where $N[r+w]$ is defined in $(2.11)$.

3. The static problem. If $w=\omega(x)$ is an equilibrium solution then $\omega$ must satisfy the ordinary differential equation

$$
N[r+w] \frac{d^{2}}{d x^{2}}(r+w)-\frac{1}{N[r+w]}(r+w)=0
$$

and boundary conditions

$$
\omega^{\prime}(0)=\omega(l)=0 .
$$

It is immediately evident from (3.1) that the static theory is independent of the choice of $r(x)$. Indeed, the actual equilibrium position of the film, i.e. $r+w$, can be treated as the dependent variable. Thus introducing the notation

$$
V(x)=r(x)+\omega(x)
$$

Eq. (3.1) becomes

$$
N[V] V^{\prime \prime}-\frac{1}{N[V]} V=0
$$

and $V$ must satisfy the boundary conditions

$$
V^{\prime}(0)=0, \quad V(l)=a .
$$


The equation (3.4) is nonlinear. However, since $N(V)$ does not depend on $x$, the equation is easily solved. In fact, introducing the notation

$$
\left(\frac{B}{l}\right)^{2}=\frac{1}{N(V)^{2}}
$$

the solution of (3.4) satisfying the boundary conditions (3.5) is

$$
V=\frac{a \cosh (B x / l)}{\cosh B}
$$

where $B(B>0)$ is to be chosen so that (3.6) is satisfied. If (3.7) is introduced into (3.6) there is no difficulty in showing that $B$ must be a solution of the algebraic equation

$$
\frac{a}{l} B=\cosh B
$$

This equation may have one, two, or no roots depending on the value of $a / l$.

Let $B=B_{0}$ be the (unique) solution of

$$
B_{0} \tanh B_{0}=1 .
$$

If

$$
a / l=\sinh B_{0}=c
$$

then Eq. (3.8) has the unique solution $B=B_{0}$. If $a / l>c$ there are two solutions which we denote by $B_{-}$and $B_{+}$where $B_{-}<B_{0}<B_{+}$. If $a / l<c$ there are no solutions.

4. Stability. In Sec. 3 it was shown that if $a / l>c$ then Eq. (2.12) has two equilibrium solutions

$$
\omega_{ \pm}(x)=\frac{a \cosh \left(B_{ \pm} x / l\right)}{\cosh B_{ \pm}}-r(x) .
$$

It is convenient to choose one of these equilibria as the reference surface $r(x)$. Thus we choose

$$
r(x)=\frac{a \cosh (B x / l)}{\cosh B}
$$

where $B$ is either $B_{+}, B_{-}$or, in the particular case where $a / l=c, B=B_{0}$. The function $r(x)$ will satisfy the differential equation

$$
r^{\prime \prime}-\left(\frac{B}{l}\right)^{2} r=0
$$

and boundary conditions

$$
r^{\prime}(0)=0, \quad r(l)=a .
$$

It follows of course that $w(x, t)=0$ is an equilibrium solution of (2.12). Indeed, with the above choice for $r(x)$ Eq. (2.12) becomes

$$
\rho\left(\frac{B}{l}\right) r^{2} w_{t t}-K N[r+w] w_{x x}+\frac{K}{N[r+w]} w=K\left(\left(\frac{B}{l}\right)^{2} N[r+w]-\frac{1}{N[r+w]}\right) r .
$$


It is easily verified that the right side of (4.5) vanishes when $w=0$

Our interest is in the linear stability of $w=0$ for the various possible choices of $r(x)$, i.e. with $B=B_{+}, B=B_{-}$, or $B=B_{0}$ (if $a / l=c$ ). The linearization is somewhat clearer if we introduce the notation

$$
\alpha[r+w]^{2}=\int_{0}^{l}(r+w)^{2} d x, \quad \beta[r+w]^{2}=\int_{0}^{l}\left(1+\left(r^{\prime}+w_{x}\right)^{2}\right) d x
$$

In this notation Eq. (4.5) becomes

$$
\begin{aligned}
\rho\left(\frac{B}{l}\right) r^{2} \alpha[r+w] \beta[r+w] & w_{t t}-K \alpha[r+w]^{2} w_{x x} \\
& +K \beta[r+w]^{2} w=K\left(\left(\frac{B}{l}\right)^{2} \alpha[r+w]^{2}-\beta[r+w]^{2}\right) r
\end{aligned}
$$

If only terms linear in $w$ are retained in (4.7) we find that

$$
\begin{aligned}
\rho\left(\frac{B}{l}\right) r^{2} \alpha[r] \beta[r] w_{t t} & -K \alpha[r]^{2} w_{x x}+K \beta[r]^{2} w \\
& =K\left(\left(\frac{B}{l}\right)^{2} \alpha[r]^{2}-\beta[r]^{2}+2\left(\frac{B}{l}\right)^{2} \int_{0}^{l} r w d x-2 \int_{0}^{l} r^{\prime} w_{x} d x\right) r
\end{aligned}
$$

where

$$
\begin{gathered}
\alpha[r]^{2}=\int_{0}^{l} r^{2} d x, \\
\beta[r]^{2}=\int_{0}^{l}\left(1+\left(r^{\prime}\right)^{2}\right) d x=\left(\frac{B}{l}\right)^{2} \int_{0}^{l} r^{2} d x=\left(\frac{B}{l}\right)^{2} \alpha[r]^{2} .
\end{gathered}
$$

In addition, an integration by parts shows that

$$
\int_{0}^{l} r^{\prime} w_{x} d x=-\int_{0}^{l} r^{\prime \prime} w d x=-\left(\frac{B}{l}\right)^{2} \int_{0}^{l} r w d x .
$$

Thus (4.8) becomes

$$
\rho\left(\frac{B}{l}\right)^{2} r^{2} w_{t t}-K w_{x x}+K\left(\frac{B}{l}\right)^{2} w=\frac{4 r}{\alpha[r]^{2}}\left(\frac{B}{l}\right)^{2} \int_{0}^{l} r w d x .
$$

In order to decide whether $w=0$ is a stable or unstable equilibrium we must decide whether solutions of (4.10) are bounded or growing. We look for solutions of (4.10) of the form

$$
w(x, t)=T(t) \omega(x)
$$

Supposedly the general solution of $(4.10)$ could be constructed by an appropriate superposition of such solutions. Placing (4.11) into (4.10), we find that $T(t)$ and $\omega(x)$ must satisfy 
the ordinary differential equations

$$
\begin{gathered}
\rho\left(\frac{B}{l}\right)^{2} T+\lambda T=0, \\
\omega^{\prime \prime}+\left(\frac{\lambda}{K} r^{2}-\left(\frac{B}{l}\right)^{2}\right) \omega+\frac{4 r}{\alpha[r]^{2}}\left(\frac{B}{l}\right)^{2} \int_{0}^{l} r \omega d x=0
\end{gathered}
$$

where $\lambda$ is the separation constant and plays the role of an eigenvalue in (4.13). $\omega$ must satisfy the boundary conditions

$$
\omega^{\prime}(0)=\omega(l)=0 .
$$

If all of the eigenvalues $\lambda_{j}$ of (4.13) are positive then the corresponding solutions of (4.12) are bounded and the equilibrium is stable. If any of the $\lambda_{j} \leq 0$ then (4.12) has growing solutions and the equilibrium is unstable. Thus the object is to determine the sign of the eigenvalues $\lambda_{j}$. It is convenient to begin by asking if $\lambda=0$ is an eigenvalue, i.e. does

$$
\omega^{\prime \prime}-\left(\frac{B}{l}\right)^{2} \omega+\frac{4 r}{\alpha(r)^{2}}\left(\frac{B}{l}\right)^{2} \int_{0}^{l} r \omega d x=0
$$

have a nontrivial solution satisfying (4.14)? Eq. (4.15) can be rewritten as the integral equation

$$
\omega(x)=\frac{2 \int_{0}^{l} r \omega d x}{\alpha(r)^{2}}\left((B \tanh B) r-x r^{\prime}\right)
$$

where we have used the fact that $r(x)$ is a solution of (4.3). It is a consequence of (4.16) that

$$
\int_{0}^{l} r \omega d x=\frac{2 \int_{0}^{l} r \omega d x}{\alpha(r)^{2}}\left(B \tanh B \int_{0}^{l} r^{2} d x-\int_{0}^{l} x r r^{\prime} d x\right) .
$$

Thus (4.16) will have a nontrivial solution if $B$ satisfies

$$
1=\frac{2}{\alpha(r)^{2}}\left((B \tanh B) \alpha(r)^{2}-\int_{0}^{l} x r r^{\prime} d x\right)=f(B) .
$$

A calculation shows that $B$ satisfies (4.18) iff $B$ satisfies (3.10). Thus the problem (4.13) and (4.14) has $\lambda=0$ as an eigenvalue iff $B=B_{0}$ which requires that $a / l=c$ (cf. Sec. 3). In this case the equilibrium is unstable, i.e. the equilibrium (4.2) with $B=B_{0}$ is unstable.

If $\lambda$ is less than the smallest eigenvalue of

$$
\omega^{\prime \prime}+\left(\frac{\lambda}{K} r^{2}-\left(\frac{B}{l}\right)^{2}\right) \omega=0
$$

with $\omega$ satisfying (4.14), Eq. (4.13) can be rewritten as the integral equation

$$
\omega(x)=\frac{4 \int_{0}^{l} r \omega d x}{\alpha[r]^{2}}\left(\frac{B}{l}\right)^{2} \int_{0}^{l} G(x, \xi ; B, \lambda) r(\xi) d \xi
$$

where $G(x, \xi ; B, \lambda)$ is the Green's function for Eq. (4.19). Eq. (4.20) reduces to (4.16) when 
$\lambda=0$. If $\omega(x)$ is a nontrivial solution of (4.20) then it follows that

$$
1=\frac{4}{\alpha[r]^{2}}\left(\frac{B}{l}\right)^{2} \int_{0}^{l} r(x) \int_{0}^{l} G(x, \xi ; B \lambda) r(\xi) d \xi d x=f_{1}(B, \lambda)
$$

which reduces to $(4.18)$ when $\hat{\lambda}=0$, i.e.

$$
f_{1}(B, 0)=f(B)
$$

(cf. (4.18)). The qualitative behavior of $G(x, \xi ; B, \lambda)$ as a function of $\lambda$ is easily described. It may be shown that $G(x, \xi ; B, \lambda) \geq 0$ and that $G(x, \xi ; B, \lambda)$ is an increasing function of $\lambda$ if $-\infty<\lambda / K \leq(B / l)^{2}$ (cf. Lemmas 2.2 and 2.3 in [10]). Thus if $\lambda<0$ we see that $G(x, \xi ; B, \lambda)<G(x, \xi ; B, 0)$ so that $f_{1}(B, \lambda)<f_{1}(B, 0)=f(B)$. Thus solutions of (4.2) with $\lambda<0$ all have $B>B_{0}$. Since $B_{-}<B_{0}$ we conclude that values $B=B_{-}$cannot correspond to negative eigenvalues, i.e. equilibria (4.2) with $B=B_{\text {- }}$ are stable.

\section{REFERENCES}

[1] R. W. Dickey, Dynamic behavior of soap films, Quart. Appl. Math. 24, 97-106 (1966)

[2] A. Foppl, Vorlesungen über technische Mechanik, Bd. 5, G. Teubner, Leipzig 1905

[3] E. Bromberg and J. J. Stoker, Non-linear theory of curved elastic sheets, Quart. Appl. Math. 3, 246-265 (1945/46)

[4] E. Reissner, Rotationally symmetric problems in the theory of thin elastic shells, 3rd U.S. Nat. Congress of Appl. Mechs., 51-69, 1958

[5] S. Woinowsky-Krieger, The effect of axial force on the vibration of hinged bars, J. Appl. Mechs. 17, 35-36 (1950)

[6] R. Narasimha, Non-linear vibrations of an elastic string, J. Sound Vib. 8, 134-146(1968)

[7] R. W. Dickey, Dynamic behavior of cylindrical membranes, Int. J. Non-Linear Mech. 6, 729-734 (1971)

[8] G. Bliss, Calculus of variations, Open Court Pub., La Salle Ill., 1925

[9] R. Courant and D. Hilbert, Methods of mathematical physics, V. I. Interscience Publishers, New York, 1962

[10] R. W. Dickey, The suspension bridge deflection equations, J. Math. Anal. Appls. 24, 202-211 (1968)

[11] R. W. Dickey, Infinite systems of nonlinear oscillation equations related to the string, Proc. Amer. Math. Soc. 23, 459-468 (1969)

[12] R. W. Dickey, Free vibrations and dynamic buckling of the extensible beam, J. Math. Anal. Appls. 29, 443-454 (1970) 